\title{
Control of expression patterns of anthocyanin structural genes by two loci in the common morning glory
}

\author{
Peter Tiffin, Richard E. Miller, and Mark D. Rausher* \\ Department of Zoology, Box 90325, Duke University, \\ Durham, North Carolina 27708-0325, U.S.A.
}

(Received 28 October 1997, accepted 12 May 1998)

\begin{abstract}
Two loci producing white flowers have been identified in the common morning glory, Ipomoea purpurea. At the $W$ locus, $w w$ individuals produce flowers with white corollas and pigmented rays, while at the $A$ locus, $a a$ individuals produce flowers with white corollas, and $a^{*} a^{*}$ individuals produce variegated corollas. To determine whether these two loci correspond to regulatory or structural genes, six structural genes required for anthocyanin synthesis were cloned and their expression pattern was examined in genotypes with white and pigmented flowers. In $w w$ flower buds, expression of all six structural genes was greatly reduced or eliminated, indicating that the $W$ locus encodes a regulatory gene. By contrast, genotype at the $A$ locus did not affect expression of any of the structural genes, suggesting that the $A$ locus may encode one of those structural genes. An evolutionary comparison of structural gene regulation in maize, snapdragons, petunias, and morning glories suggests that regulatory control of the anthocyanin pathway is evolutionarily labile.
\end{abstract}

\section{INTRODUCTION}

The flavonoid pathway, and in particular its anthocyanin branch, has served as a paradigm for understanding gene regulation in plants (Dooner et al., 1991; van der Meer et al., 1993; Holton and Cornish, 1995). In addition, recent evolutionary studies suggest that this pathway may also serve as a model for elucidating the evolution of plant metabolic pathways at the molecular level (Koes et al., 1994; Shirley, 1996). In both of these areas, however, investigation has concentrated primarily on three species: maize (Zea mays), petunia (Petunia hybrida), and snapdragon (Antirrhinum majus), primarily because until recently it was only in these three species that most or all of the six known structural genes required for anthocyanin production have been cloned and characterized. While investigations of these three species have already led to some important generalizations, such as the conservation of some regulatory genes across a wide range of taxa (Goodrich et al., 1992; Quattrocchio et al., 1993), further generalizations regarding both regulation and evolution will be more robust if these processes can be examined in additional species. For this reason, we have undertaken an attempt to characterize the anthocyanin pathway structural genes in a species of morning glory, Ipomoea purpurea (Convolvulaceae).

Like its close relative, the Japanese morning glory I. nil, I. purpurea exhibits a variety of genetic variants that differ in flower color (Ennos and Clegg, 1983; Epperson and

\footnotetext{
* Corresponding author.
}

Clegg, 1987, 1988, 1992). In particular, mutations at two loci produce white flowers rather than the usual blue or pink flowers. At the $W$ ("white") locus, individuals that are homozygous $W W$ produce normally pigmented flowers, while $w w$ individuals produce flowers with white corollas and pigmented rays and $W w$ individuals produce lightly pigmented flowers. There are no apparent pleiotropic effects of the $w$ allele on pigmentation in other tissues (e.g., no effects on stem or leaf pigmentation). At the $A$ ("albino") locus, the flowers of $A A$ individuals are normally pigmented, while those of $a a$ plants have a completely white corolla without pigmented rays. Heterozygous $A a$ individuals produce lightly pigmented flowers. By contrast with the $W$ locus, homozygous $a a$ individuals exhibit pleiotropic effects on stem pigmentation: $A A$ individuals have red to brown stems, whereas aa individuals have green stems. In addition, a third, unstable allele, $a^{*}$, has been characterized at the $A$ locus. Individuals that are $a^{*} a^{*}$ exhibit variegation of flowers and stems. The rates of mutation to and from $a^{*}$ suggest that the unstable nature of the pigment pattern associated with the $a^{*}$ allele results from the presence of a transposable element (Epperson and Clegg, 1992).

The lack of pleiotropic effects of the $W$ locus, along with the production of pigmentation in rays of $w w$ individuals, suggests that this locus may regulate the expression of one or more of the structural genes needed for pigment production. In these properties, this locus is similar to the Delila locus of Antirrhinum (Goodrich et al., 1992) and the An2 and An4 loci of Petunia (Quattrocchio et al., 1993), regula- 
tory loci apparently homologous to the $R$ and $C 1$ family of transcriptional activators of maize. By contrast, the complete elimination of pigmentation from both flowers and some non-floral tissue suggests that the $A$ locus may correspond to a structural gene that is expressed in several tissues. A similar phenotypic pattern is seen in $I$. nil due to inactivation of the $D F R$ gene by a transposable element (Inagaki et al., 1994,1996).

In this report, we attempt to determine whether the $W$ and $A$ loci correspond to regulatory or structural loci. To do so, we describe the cloning and characterization of the genes chalcone synthase $(\mathrm{CHS})$, chalcone isomerase $(\mathrm{CHI})$, flavanone 3-hydroxylase $(F 3 H)$, dihydroflavonol 4-reductase $(D F R)$, anthocyanin synthase (ANS), and UDP glucose: flavonoid 3-O-glucosyltransferase (UF3GT) from a cDNA library of $I$. purpurea floral-bud tissue. We also describe the expression patterns of these genes, which indicates that the $W$ locus regulates the expression of all six structural genes in flower buds, whereas the $A$ locus does not affect the expression pattern of these genes. Finally, we discuss the implications of these results for the evolution of regulation of the anthocyanin pathway in angiosperms.

\section{MATERIALS AND METHODS}

Isolation of structural genes. Structural genes were isolated from a cDNA library constructed from the distal half of flower buds of $I$. purpurea using the ZAP cDNA synthesis kit (Stratagene). Our general strategy for isolation was to use PCR to obtain a small fragment of a gene, then use this fragment as a probe of the library to isolate the entire cDNA for the gene. Degenerate PCR primers were designed based on conserved sequences in the appropriate genes, as determined by comparing sequences reported in GenBank, and are listed in Table 1. PCR reactions were performed under one of two conditions, depending on the particular gene (Table 1). Fragments obtained were sequenced to verify homology to the gene sought and hence appropriateness for use as a probe. For each gene, approximately 300,000 plaques were probed and one to ten highly hybridizing clones were isolated and sequenced. For isolating the UF3GT gene, we probed the cDNA library with a fragment of the homologous snapdragon gene. This fragment was produced using the PCR primers indicated in Table 1 with the entire snapdragon gene (supplied by Cathie Martin) as a template.

Analysis of expression patterns. We examined the expression patterns of the six structural genes in the distal portion of I. purpurea flower buds using RNA (Northern) blotting. Total RNA was extracted from the distal half of flower buds using a Promega isolation kit. For each gene, a 500-1000 bp fragment was used as a probe for its transcript. To make the blots, approximately $15 \mu \mathrm{g}$ of total RNA was subjected to denaturing electrophoresis (Sambrook et al., 1989), then transferred to a nylon membrane (Hybond $\mathrm{N}^{+}$, Amersham). Probing was conducted at $65^{\circ} \mathrm{C}$, with washing at $60^{\circ} \mathrm{C}$, and probed membranes were read using a phosphorimager. We also probed blots with a fragment of the I. purpurea $18 \mathrm{~S}$ ribosomal RNA gene as a control for RNA loading.

Strains examined were from our collection of inbred lines and included: line J (pigmented); line N ( $a a$, white flowers); line $\mathrm{W}\left(a^{*} a^{*}\right.$, with mostly white flowers and minimal flecking); and line $\mathrm{P}(w w)$. For the ww genotype, bud tips were dissected and the pigmented ray tissue removed before RNA extraction. As a control, pigmented ( $W W$ ) buds were also dissected and extracted in a similar way. For the remaining genotypes, including $W W$, RNA was extracted from undissected bud tips.

Table 1. Accession numbers and PCR primers and conditions used to obtain gene fragments used as probes

\begin{tabular}{|c|c|c|c|}
\hline Gene & $\begin{array}{c}\text { Accession } \\
\text { No. }\end{array}$ & PCR Primers & PCR Conditions ${ }^{\mathrm{a}}$ \\
\hline \multirow[t]{2}{*}{ CHS } & U74082 & ATGATGTA[TC]CA[AG]CA[AG]GGNTG & 1 \\
\hline & & GCNGG[AG]TGNGC[ACG]ATCCA[AG]AA & \\
\hline \multirow[t]{2}{*}{ CHI } & AF028238 & TT[AC]CTNG[GC]NGGNGCNGGN & 2 \\
\hline & & ATNGANGCNCCNGGNGG[AG]AA & \\
\hline \multirow[t]{2}{*}{ F3H } & U74081 & GTNTCNAG[TC]CA[TC]CTNCA[AG]GG & 2 \\
\hline & & TT[TC]TG[AG]TCCAT[AG]TCNAC[AG]CA & \\
\hline \multirow[t]{2}{*}{ DFR } & AF028601 & $\mathrm{GA}[\mathrm{AG}] \mathrm{AA}[\mathrm{TC}] \mathrm{GA}[\mathrm{AG}] \mathrm{GTNAT}[\mathrm{ACT}] \mathrm{AA}[\mathrm{AG}] \mathrm{CC}$ & 1 \\
\hline & & GG[ATG]AT[ATG]AT[AG]CT[ATG]AT[AG]AA[AG]TC & \\
\hline \multirow[t]{2}{*}{ ANS } & AF028602 & GA[AG]TGGGA[AG]GA[TC]TA[TC]TT[TC]TT & 2 \\
\hline & & TCNGT[AG]TGNGC[TC]TCNACNCC & \\
\hline \multirow[t]{2}{*}{ UF3GT } & AF028237 & AAAGCCCTACCACAAGGATT & 1 \\
\hline & & ATCTCTAACCCTAACCCCAA & \\
\hline
\end{tabular}

\footnotetext{
${ }^{\text {a }}$ Condition 1: 35 cycles of $94^{\circ} \mathrm{C}$ for $1 \mathrm{~min}$., $37^{\circ} \mathrm{C}$ for $1 \mathrm{~min}$., $72^{\circ} \mathrm{C}$ for $30 \mathrm{sec}$.

Condition 2: 15 cycles of $94^{\circ} \mathrm{C}$ for $1 \mathrm{~min}$., $37^{\circ} \mathrm{C}$ for $1 \mathrm{~min}$., $72^{\circ} \mathrm{C}$ for $30 \mathrm{sec}$, followed by 25 cycles of $94^{\circ} \mathrm{C}$ for $1 \mathrm{~min}$., $50^{\circ} \mathrm{C}$ for $1 \mathrm{~min}$., $72^{\circ} \mathrm{C}$ for $30 \mathrm{sec}$.
} 


\section{RESULTS}

Isolation of structural genes. For the genes $C H I, F 3 H$, $D F R$, and ANS we were able to obtain complete coding sequences, as indicated by the presence of the expected start codon and a poly-A tail. We obtained a truncated $C H S$ gene, the sequence of which was identical to that of the $C H S-E$ gene subsequently reported by Fukada-Tanaka et al. (1997), except that it lacked $249 \mathrm{bp}$ at the 3 ' end of the sequence, though it included a poly-A tail. To date we have obtained only a partial sequence for UF3GT. This sequence is 1512 bp long and contains a poly-A tail, but lacks approximately $115 \mathrm{bp}$ from the 5' end, compared to the snapdragon UF3GT sequence. Sequences of these genes have been deposited in GenBank and accession numbers are listed in Table 1.

Generally, the nucleotide and inferred amino acid sequences of the genes we have isolated exhibit high similarity to the sequences of the corresponding petunia genes, and very high similarity (greater than $95 \%$ ) to genes isolated from the closely related I. nil (Table 2). Moreover, this high similarity exists along the entire length of each gene. In the case of $\mathrm{CHI}$, where the percent identity with Petunia is lower than $70 \%$, comparisons of the sequences among other species (e.g., petunia vs. snapdragon, proportion identity of amino acid sequences $=0.627$, vs. 0.653 for I. purpurea and snapdragon) also exhibit similarly reduced

Table 2. Degree of homology between isolated Ipomoea purpurea sequence and homologous sequence from other species

\begin{tabular}{lcccc}
\hline \hline Gene & Ipomoea nil & Petunia & Antirrhinum & Zea \\
\hline CHS & 0.959 & 0.856 & 0.845 & 0.801 \\
& 0.955 & 0.766 & 0.717 & 0.713 \\
CHI & NA & 0.661 & 0.613 & 0.569 \\
& & 0.688 & 0.653 & 0.618 \\
F3H & 0.978 & 0.814 & 0.675 & 0.735 \\
& 0.979 & 0.757 & 0.656 & 0.700 \\
DFR & 0.953 & 0.717 & 0.629 & 0.567 \\
& 0.964 & 0.735 & 0.663 & 0.590 \\
ANS & NA & 0.770 & 0.740 & 0.508 \\
& & 0.725 & 0.702 & 0.572 \\
UF3GT & NA & NA & 0.423 & 0.270 \\
& & & 0.528 & 0.451 \\
\hline
\end{tabular}

Top number indicates fraction identity in amino-acid composition, while bottom number indicates fraction identity in nucleotide composition. Comparisons are with $C H S-E$ and DFR-B genes from Ipomoea nil, CHS-A gene from Petunia, and $C H S C 2$ gene from Zea. NA: sequence not available for comparison with $I$. purpurea sequence. Accession numbers for compared sequences: (1) Ipomoea nil CHS-AB001819, F3H-D83041, DFR-AB006792; (2) Petunia CHS-X14591, CHI-Y00852, F3H-X60512, DFR-X79723; (3) Antirrhinum CHS-X03710, CHI-M68326, DFR-X15536; (4) Zea CHSX60205, CHI-Z22760, F3H-U04434, ANS-X55314, UF3GTX13502. Antirrhinum sequences for F3H, ANS, and UF3GT were provided by Cathie Martin. Petunia ANS and Zea DFR sequences were obtained from the literature. homology, indicating more rapid divergence of this gene. As expected, the proportion identity is lower, but still appreciable, when I. purpurea is compared with the more distantly related snapdragon and maize.

For UF3GT, no comparison is possible with either I. nil or petunia, since cloning of this gene has not been reported for these species. Homology of our clone is lower with snapdragon and maize UF3GT than in similar comparisons with the other genes; but the sequence identity between snapdragon and maize UF3GT (0.434 for nucleotide sequence, 0.305 for amino acid sequence) is similar to that seen between I. purpurea and maize, as would be expected because the snapdragon and I. purpurea lineages have been separated the same amount of time from the maize lineage. As in the case of $\mathrm{CHI}$, these results suggest that UF3GT has an elevated rate of evolutionary change, compared to the other anthocyanin structural genes.

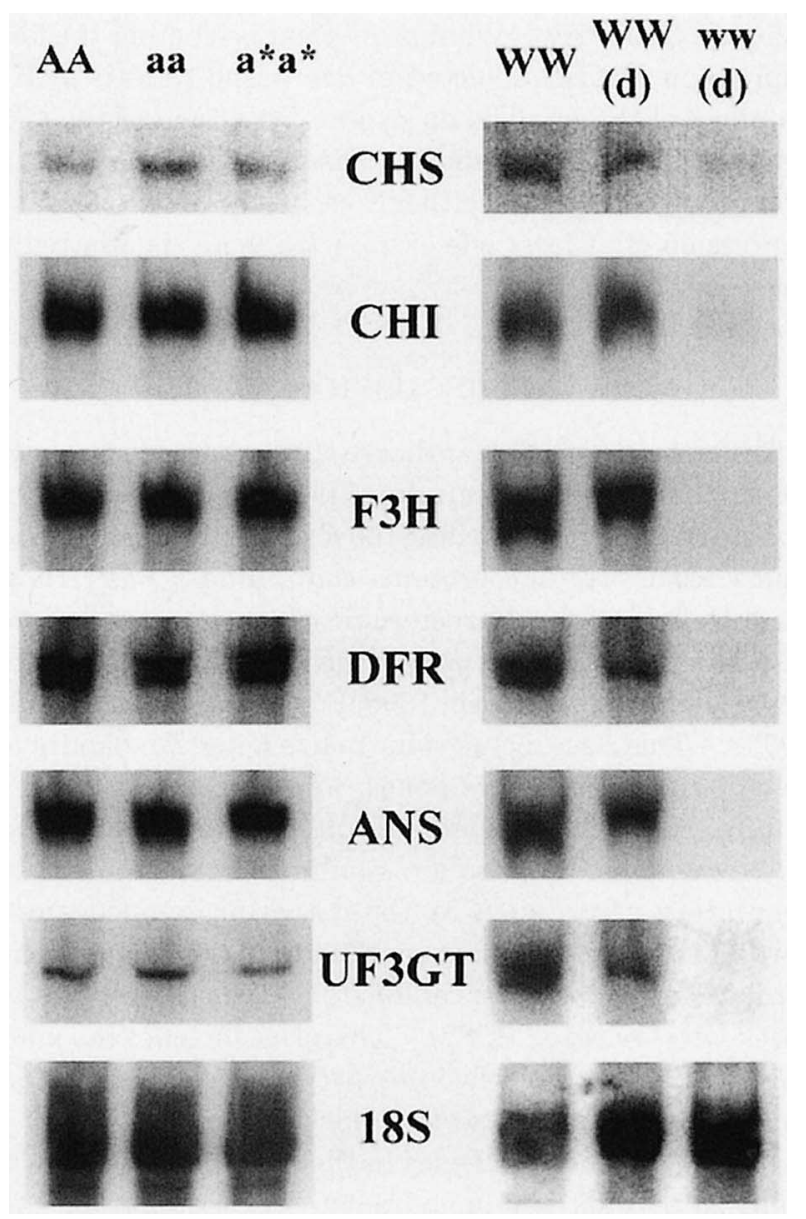

Fig. 1. RNA gel blot analysis of anthocyanin structural genes of Ipomoea purpurea. Total RNA $(15 \mu \mathrm{g})$ from the distal half of flower buds were hybridized with the indicated cDNA probe as described by Sambrook et al. 1989. Genotypes corresponding to individual lanes are indicated at top. WW(d) and ww(d) lanes correspond to RNA extracted from bud tips with the pigmented ray tissue dissected out, while all other lanes represent RNA from entire bud tips. 18S RNA fragment is control for RNA loading. 
Analysis of expression patterns. Expression of each of the six structural genes was comparable for the $A A, a a$, and $a^{*} a^{*}$ genotypes (Fig. 1). By contrast, all six genes had greatly reduced expression in the $w w$ genotype. CHS and CHI exhibited faint expression, while for the other genes no transcript was detected. The general intensity of $\mathrm{CHS}$ expression in all genotypes appears lower than that of the other genes. We suspect that the reason for this reduced intensity is that the primary $C H S$ transcript expressed in corolla tissue (corresponding to the distal portion of the bud we have examined) is $C H S-D$, while the probe we used is $C H S-E$. While both genes are expressed in corolla tissue, CHS-D predominates, constituting approximately $85 \%$ of the CHS transcripts (Fukada-Tanaka et al., 1997). Moreover, the nucleotide sequence identity between $C H S-D$ and $C H S-E$ is only approximately $80 \%$, which could reduce the specificity of the $C H S-E$ probe to the predominant $C H S-D$ transcript, thus weakening the signal. Fukada-Tanaka et al. (1997) report such signal weakening in their probing of their cDNA library. What is not clear is whether the faint expression of $C H S$ detected in $w w$ tissue reflects lack of an effect of the $w$ allele on expression of one of the CHS genes, or simply represents a baseline "leakiness" in the expression of $C H S$. In either case, however, it is clear that expression of at least one of the $C H S$ genes is affected by the $W$ locus.

\section{DISCUSSION}

The current model of anthocyanin structural gene regulation acknowledges the role of two classes of transcriptional activators. One class, the $R$ family, consists of genes that encode myc-like proteins containing a basic HelixLoop-Helix domain characteristic of other transcriptional activators such as Max and MyoD1 (van der Meer et al., 1993; Holton and Cornish, 1995; Purugganan and Wessler, 1994 ). This class includes the maize $R$ and $B$, snapdragon Delila, and petunia An4 genes. The second class, the $C$ family, consists of genes encoding proteins containing an $m y b$-like binding domain similar to that of eukaryotic regulatory genes such as the $c-m y b$ proto-oncogene of vertebrates and the glabrous gene that controls trichome formation in Arabidopsis and Petunia (van der Meer et al., 1993; Paz-Ares et al., 1987). This class includes the maize $C 1$ and $P l$, the snapdragon myb305 and the petunia An2 genes. In maize, these two classes of genes appear to regulate all structural genes in the anthocyanin pathway, while in petunia and snapdragon they regulate only the three or four most downstream genes (e.g., $F 3 H, D F R$, ANS, and UF3GT; Holton and Cornish, 1995). This difference in regulation has led Koes et al. (1994) to outline a simple model of the evolution of the anthocyanin pathway in angiosperms involving at least one episode of evolutionary loss of regulatory control over $\mathrm{CHS}$ and $\mathrm{CHI}$, in addition to loss of regulatory control of $F 3 H$ in the petunia lineage.
The pattern of structural gene expression, as controlled by the $W$ locus in $I$. purpurea, suggests that one or more aspects of this model are incomplete. In particular, the elimination of expression of all six structural genes in $w w$ individuals indicates that the $W$ locus is a major regulator of anthocyanin production in flowers of $I$. purpurea. However, the set of genes regulated differs from that controlled by known regulatory genes in petunia and snapdragon. Consequently, either the $W$ locus represents a new class of anthocyanin regulatory genes or the control of structural gene expression by $m y b$ - and $m y c$-like genes is more evolutionarily labile than previously envisioned.

Which of these possibilites is correct will only be settled definitively by cloning and characterizing the $W$ locus. Nevertheless, several considerations suggest that the latter possibility is more likely. First, all anthocyanin regulatory genes that have been cloned were first characterized genetically as color mutants (van der Meer et al., 1993). It seems unlikely to us that if there are indeed additional classes of regulators, all known mutants should turn out to belong to only two classes of homologous regulators. Second, while the pattern of regulation exhibited by the $W$ locus differs from that exhibited by regulatory genes in other dicots, it is similar to that seen in maize. Thus, no novel type of regulatory control, and hence no novel class of regulatory genes, need be invoked to explain the pattern exhibited by the $W$ locus.

If, as we believe, this conclusion is correct, the pattern of gene regulation by the $W$ locus indicates that the set of genes controlled by anthocyanin regulators is evolutionarily more labile than previously suspected. The model of Koes et al. (1994) suggests that coordinated regulation of the six major anthocyanin genes evolved early in the angiosperm lineage to ensure simultaneous expression of all genes needed for pigment production in the flower. It further envisions that in one or more dicot lineages, genes upstream in the biosynthetic pathway, particularly $C H S$ and $C H I$, were removed from this common regulatory network. In particular, the minimally parsimonious tree under this model postulates a single loss of regulation of these two genes in the common lineage of petunias and snapdragons (Fig. 2c, ignoring the Ipomoea lineage). However, because this model was based on regulatory patterns observed in just three species (maize, petunia, and snapdragon), it can not be distinguished from an equally parsimonious alternative model in which absence of regulatory control of these two genes was the ancestral state (Fig. 2a, ignoring the Ipomoea lineage).

With the addition of Ipomoea to the phylogeny, it is still impossible to distinguish whether or not coordinate regulation of all six genes is ancestral in angiosperms. However, regardless of ancestral state, all minimally parsimonious phylogenetic trees require at least two evolutionary changes in the regulation of $\mathrm{CHS}$ and $\mathrm{CHI}$ (Fig. 2). Consequently, adding one additional species to the known pat- 


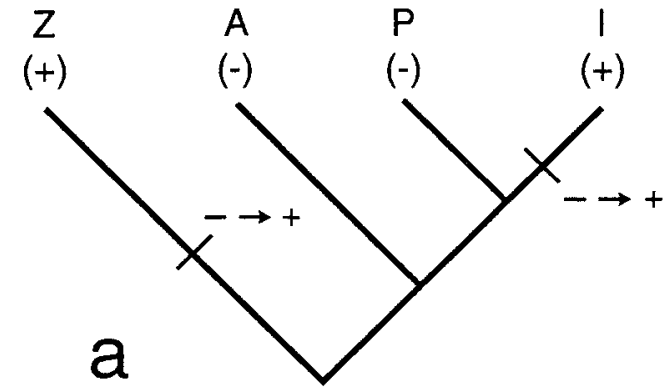

$(-)$

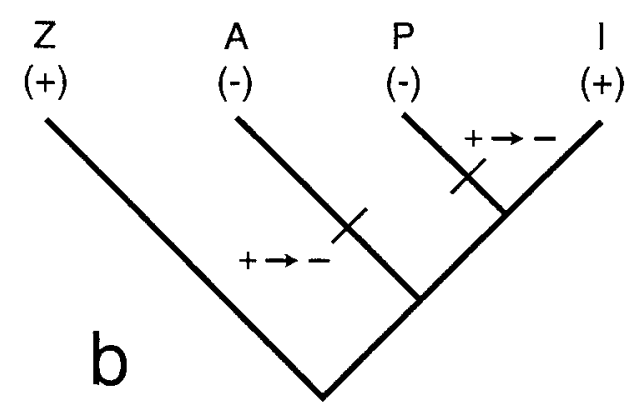

$(+)$

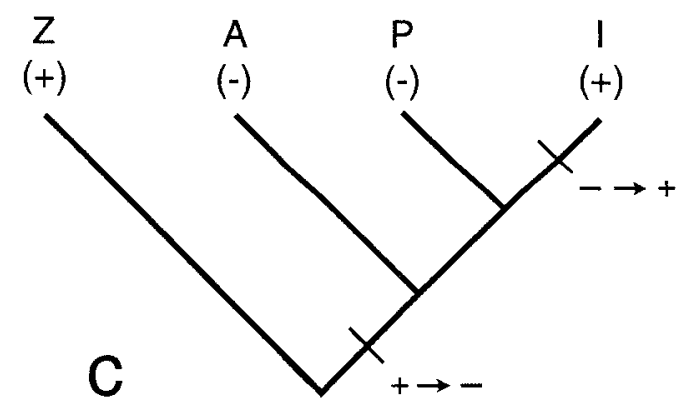

$(+)$

Fig. 2. Evolutionary transitions in regulatory control of $\mathrm{CHI}$ and $C H S$ by known or suspected $m y b$ - and $m y c$-like genes superimposed on known phylogeny (Chase et al., 1993) of the four species in which control has been examined: Zea mays (Z), Antirrhinum majus (A), Petunia hybrida (P), and Ipomoea purpurea (I). The three maximally parsimonious arrangements of transitions are shown. (+) and (-) refer to control and lack of control, respectively.

terns of regulation of $m y b$ - and $m y c$-like genes indicates that alteration of regulatory control of these two genes could not have occurred just once in angiosperm evolution. Given a minimum of two evolutionary changes in regulation of these two genes when only four taxa are examined, we suggest that examination of the pattern of regulation in other taxa will reveal even greater evolutionary lability of regulation of these and other anthocyanin genes.

Unlike the $W$ locus, the $A$ locus appears to play no role in the regulation of the six structural genes examined, suggesting that $A$ may encode a structural gene rather than a regulatory gene. The phenotypes of $a a$ and $a^{*} a^{*}$ individuals are very similar to those reported by Inagaki et al. (1994, 1996) for the Japanese morning glory, I. nil, having white $(a a)$ or variegated $\left(a^{*} a^{*}\right)$ flowers and lacking pigmentation in the stem. In the closely related $I$. nil, these phenotypes result from the insertion of the transposable element Tpn1 into an intron in the DFR-B gene. Thus, the structural gene $D F R$ seems a likely candidate for the $A$ locus in I. purpurea. Although absence of an apparent difference in $D F R$ transcript length in the the $A A, a a$, and $a^{*} a^{*}$ genotypes may seem to mitigate against this inference, cases are known in which transposon splicing yields non-functional transcripts similar in length to functional transcripts produced by gene copies lacking transposon insertions (Wessler et al., 1987). Nevertheless, we can not rule out the possibility that $A$ regulates either structural genes that produce precursors of the anthocyanin pathway, such as phenylalanine ammonia lyase $(P A L)$ (van der Meer et al., 1993), or anthocyanin processing steps such as those involving vacuolar transport (Marrs et al., 1995).

A major unanswered issue in evolutionary biology is ascertaining the relative roles of structural and regulatory genes in causing phenotypic change. Recent comparative work in animals suggests that much evolutionary change in morphology is accomplished primarily by evolutionary change at regulatory loci (Akam et al., 1994; Gerhart and Kirschner, 1997). Whether this is also true of evolution in important plant characters, including flower color, is currently unknown. In this context, however, the results presented here demonstrate that naturally occurring mutations in regulatory genes have the potential to serve as the raw material for adaptive flower color change. The genus Ipomoea consists of more than 600 species (Austin and Huáman, 1996) of vines and shrubs that exhibit a tremendous diversity of flower colors, including many white-flowered species, and thus offers an opportunity to determine whether structural or regulatory genes play the primary role in evolutionary diversification of flower color. Our cloning of the six major structural genes of the anthocyanin pathway in this genus should facilitate determination of the relative importance of regulatory and structural genes in the evolutionary diversification of this important plant character.

\section{REFERENCES}

Akam, M., Holland, P., Ingham, P., and Wray, G. (eds.). (1994) The Evolution of Developmental Mechanisms. Development (1994 Supplement): 1-241.

Austin, D. F., and Huáman, Z. (1996) A synopsis of Ipomoea (Convolvulaceae) in the Americas. Taxon 45, 3-38.

Chase, M. W., Soltis, D. E., Olmstead, R. G., Morgan, D., Les, D. H., Mishler, B. D., Duvall, M. R., Price, R. A., Hills, H. G., Qiu, Y.-L., Kron, K. A., Rettic, J. H., Conti, E., Palmer, J. D., Manhart, J. R., Sytsma, K. I., Michaels, H. J., Kress, W. J., Karol, K. G., Clark, W. D., Hendren, M., Gaut, B. S., Jansen, R. K., Kim, K-I., Wimpee, C. F., Smith, J. F., Furnier, G. R., 
Strauss, S. H., Xiang, Q.-Y., Plunkett, G. M., Soltis, P. S., Swensen, S., Williams, S. E., Gadek, P. A., Quinn, C. J., Eguiarte, L. E., Golenberg, E., Learn, G. H., Graham, S. W., Barrett, S. C. H., Daanandan, S., and Albert, V. A. (1993) Phylogenetics of seed plants: An analysis of nucleotide sequences from the plastid gene $r b c L$. Ann. MO Bot. Gard. 80, 528-580.

Dooner, H. K., Robbins, T. P., and Jorgensen, R. A. (1991) Genetic and developmental control of anthocyanin biosynthesis. Annu. Rev. Genet. 25, 173-199.

Ennos, R. A., and Clegg, M. T. (1983) Flower color variation in the morning glory, Ipomoea purpurea. J. Hered. 74, 247-250.

Epperson, B. K., and Clegg, M. T. (1987) Instability at a flower color locus in the morning glory. J. Hered. 78, 346-352.

Epperson, B. K., and Clegg, M. T. (1988) Genetics of flower color polymorphism in the common morning glory (Ipomoea purpurea). J. Hered. 79, 64-68.

Epperson, B. K., and Clegg, M. T. (1992) Unstable white flower color genes and their derivatives in the morning glory. J. Hered. 83, 405-409.

Fukada-Tanaka, S., Hoshino, A., Hisatomi, Y., Habu, Y., Hasebe, M., and Iida, S. (1997) Identification of new chalcone synthase genes for flower pigmentation in the Japanese and common morning glories. Plant Cell Physiol. 38, 754-758.

Gerhart, J., and Kirschner, M. (1997) Cells, Embryos, and Evolution. Blackwell Scientific Publ., Cambridge, MA.

Goodrich, J., Carpenter, R., and Coen, E. S. (1992) A common gene regulates pigmentation pattern in diverse plant species. Cell 68, 955-964.

Holton, T. A., and Cornish, E. A. (1995) Genetics and biochemistry of anthocyanin biosynthesis. Plant Cell 7, 1071-1083.

Inagaki, Y., Hisatomi, Y., and Iida, S. (1996) Somatic mutations caused by excision of the transposable element, Tpn1, from the $D F R$ gene for pigmentation in sub-epidermal layer of periclinally chimeric flowers of Japanese morning glory and their germinal transmission to their progeny. Theor. Appl. Genet. 92, 499-504.

Inagaki, Y., Hisatomi, Y., Suzuki, T., Kasahara, K., and Iida, S.
(1994) Isolation of a suppressor-mutator/enhancer-like transposable element, Tpn1, from Japanese morning glory bearing variegated flowers. Plant Cell 6, 375-383.

Koes, R. E., Quattrocchio, R., and Mol, J. N. M. (1994) The flavonoid biosynthetic pathway in plants: function and evolution. BioEssays 16, 123-132.

Marrs, K. A., Alfenito, M. R., Lloyd, A. M., and Walbot, V. (1995) A glutathione S-transferase involved in vacuolar transfer encoded by the maize gene Bronze-2. Nature 375, 397-400.

Martin, C., Prescott, A., Mackay, S., Bartlett, J., and Vrijlandt, E. (1991) Control of anthocyanin biosynthesis in flowers of Antirrhinum majus. Plant J. 1, 37-49.

Paz-Ares, J., Ghosal, D., Wienand, U., Peterson, P. A., and Saedler, H. (1987) The regulatory $c 1$ locus of Zea mays encodes a protein with homology to myb proto-oncogene products and with structural similarities to transcriptional activators. EMBO J. 6, 3553-3558.

Purugganan, M. D., and Wessler, S. R. (1994) Molecular evolution of the plant $R$ regulatory gene family. Genetics 138, 849854.

Quattrocchio, F., Wing, J. F., Lepen, H. T. C., Mol, J. N. M., and Koes, R. E. (1993) Regulatory genes controlling anthocyanin pigmentation are functionally conserved among plant species and have distinct sets of target genes. Plant Cell 5, 14971512.

Sambrook, J., Fritsch, E. F., and Maniatis, T. (1989) Molecular Cloning: A Laboratory Manual. 2nd ed. Cold Spring Harbor Lab., Cold Spring Harbor, NY.

Shirley, B. W. (1996) Flavonoid biosynthesis: 'new' functions for an 'old' pathway. Trends Plant Sci. 1, 377-382.

van der Meer, I. M., Stuitje, A. R., and Mol, J. N. M. (1993) Regulation of general phenylpropanoid and flavonoid gene expression. In: Control of Plant Gene Expression (ed.: D. P. S. Verma), pp. 125-155. CRC Press, New York.

Wessler, S. R., Baran, G., and Varagona, M. (1987) The maize transposable element Ds is spliced from RNA. Science 237, 916-918. 